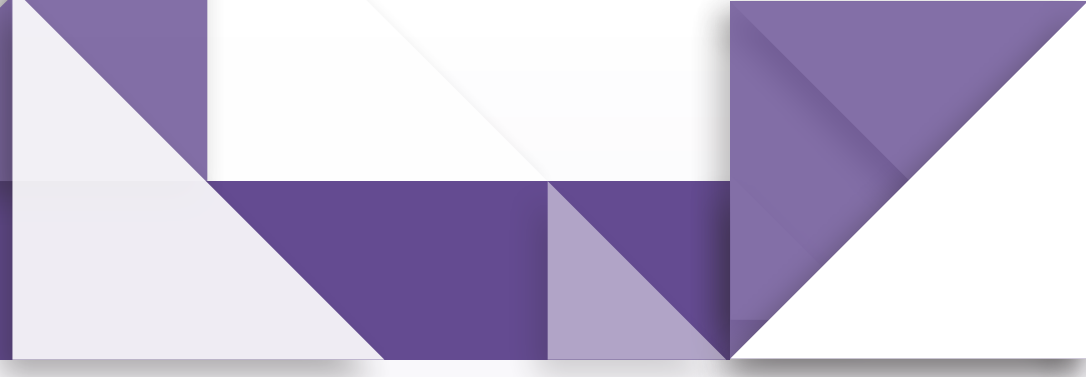

\title{
El ambiente digital en la comunicación, la actitud y las estrategias pedagógicas utilizadas por docentes 5
}

\section{The digital environment in communication, attitude and pedagogic strategics used by teachers}

\author{
ambiente digital na comunicação, na \\ atitude e nas estratégias pedagógicas \\ utilizadas pelos docentes
}

\section{Paola Lucumi Useda' \\ Martha Alexandra} González Castañeda ${ }^{2}$

\footnotetext{
1 Profesora Escuela Normal Superior Ubaté paolitalucumi@gmail.com

2 Profesora Escuela Normal Superior Ubaté marthalexgon@gmail.com
}

\section{Resumen}

El presente artículo es el estado del arte sobre el ambiente digital en la comunicación, la actitud y las estrategias pedagógicas utilizadas por docentes, a partir de tres categorías: prácticas y estrategias pedagógicas mediadas por TIC; actitud de docentes en ambientes de aprendizaje mediados por tecnología y estrategias de comunicación docente en ambientes de aprendizaje, con el objetivo de revisar los antecedentes teóricos e investigativos a partir de una revisión documental. El ejercicio condujo a concluir que en la actualidad es importante que los docentes incluyan las TIC en sus prácticas pedagógicas, muestren una actitud positiva ante los ambientes de aprendizaje e implementen el uso de estrategias innovadoras en el aula que promuevan la interacción en redes de conocimiento. Se puede afirmar que los procesos de formación de sujetos capaces de desenvolverse en esta era globalizada requieren de grandes esfuerzos de la comunidad académica por reconocer y utilizar las tecnologías de la información y la comunicación como un elemento transversal en la gestión de conocimiento; por ello, la práctica pedagógica debe ubicarse en senderos propuestos por las nuevas tecnologías, para lo cual se requiere de un trabajo sistemático hacia la comprensión de las nuevas relaciones entre docentes y estudiantes, lo que a su vez, implica el desarrollo de competencias para el aprendizaje colaborativo y la interacción. Otra importante característica que vale la pena recoger aquí es que la comunicación como acto humano es

5 Artículo de investigación científica. Estado del Arte, producto del programa "Representación ontológica hipermedial en línea para el aprendizaje significativo". Proyecto: "Efecto del ambiente digital SIMAS en la comunicación, la actitud y las estrategias pedagógicas utilizadas por docentes: validación de dos áreas y niveles del sistema educativo". 2014. Cofinanciado por Colciencias; Ministerio de Educación Nacional (MEN); Corporación Internacional de Redes de Conocimiento, ICONK; Escuela Normal Superior de Ubaté (ENSU); Fundación de Educación Superior Nueva América (Fesna); e, I3net. Código 5880-578-36127. Fecha de inicio: 30-12-2013. Fecha de terminación: 30-04-2016. Bogotá - Ubaté. 
la única que permite el reconocimiento del otro, de la diversidad, de la multiculturalidad, de la vivencia de la alteridad, como ejes fundamentales de las prácticas pedagógicas de los maestros y en general de la vida. La comunicación como una capacidad exclusivamente humana desde la reflexión, la construcción de conocimiento y pensamiento, ha permitido evidenciar el boom de las tecnologías en el mundo globalizado, encontrando otras formas de comunicación, difusión y construcción de saberes. Este proyecto pretende fortalecer procesos comunicativos que sustenten y den validez a la formación de saberes con el uso de las TIC en las prácticas pedagógicas de los maestros a través de la conformación de redes y comunidades de aprendizaje.

\title{
Palabras clave:
}

Comunicación docente, prácticas pedagógicas, estrategias pedagógicas, actitud docente, tecnologías de la información y la comunicación (TIC).

\begin{abstract}
This article is the state of the art on the effects that generates the digital environment in the communication, the attitude and the pedagogical strategies used by teachers, in three categories: instructional strategies and pedagogical practices used by teachers mediated by ICT, attitude of teachers in learning environments mediated by technology and communication strategies in teaching learning environments, with the purpose of reviewing the theoretical background and research from a documentary review. The exercise led to conclude that in the present, it is important that teachers include ICT in their teaching practices, showing a positive attitude to learning environments and implementing the use of innovative strategies in the classroom that promote interaction on knowledge networks. It can be said that the processes of formation of subjects able to thrive in this globalized era, requires a major effort of the academic community by recognizing and using information and communication technologies as a transversal element in the management of knowledge; therefore, the pedagogical practice must be located in paths proposed by the new technologies, which requires a systematic work toward understanding the new relationships between teachers and students, which in turn, involves the development of competences for collaborative learning and interaction. Another important feature that it is worth collecting here is that the communication as human act is the only one that allows the recognition of the other, for diversity, multiculturalism, the experience of otherness, as fundamental axes of the pedagogical practices of teachers in general and of life. Communication as an exclusively human capacity since the reflection, the construction of knowledge and thought, has not revealed the boom of the technologies in the globalized world, finding other forms of communication, dissemination and construction of knowledge. This project aims to strengthen communicative processes that sustain and give validity to the formation of knowledge with the use of ICT in the pedagogical practices of teachers through the formation of networks and learning communities.
\end{abstract}

\section{Keywords:}

Teacher communication, pedagogical practices, teaching strategies, teachers' attitudes, information and communication technologies (ICT).

\section{Resumo}

Este artigo, é o estado do arte sobre o ambiente digital na comunicação, na atitude e nas estratégias pedagógicas utilizadas pelos docentes, a partir de três categorias: primeira, práticas e estratégias pedagógicas utilizadas pelos docentes por meio da TIC, segunda, a atitude dos docentes em ambientes de aprendizagem por meio da tecnologia, e por último, as estratégias de comunicação docente em ambientes de aprendizagem, com o objetivo de revisar os antecedentes teóricos e investigativos a partir de uma revisão documental. $\bigcirc$ exercício deixou como conclusão que atualmente é importante que os docente incluam as TICS nas suas práticas pedagógicas, mostrando 
uma atitude positiva ante os ambientes de aprendizagem instaurando o uso de estratégias inovadoras na aula que fomente na interação nas redes do conhecimento. Pode afirmar-se que os processos de formação de sujeitos capazes de desenvolver-se nesta era globalizada, requer de enormes esforços da comunidade acadêmica por reconhecer e utilizar as tecnológicas da informação e comunicação como um elemento transversal na gestão do conhecimento, por isso, a prática pedagógica deve localizar-se nos caminhos propostos pelas novas tecnologias, que requer de um trabalho sistemático para a compreensão das novas relações entre os docentes e os alunos, que por sua vez implica o desenvolvimento de competências para a aprendizagem colaborativo e a interação. Outra importante característica vale a pena recolher aqui, é que a comunicação como ato humano é a única que permite o reconhecimento do outro, da diversidade, da multiculturalidade, as vivências da alteridade, como eixos fundamentais das práticas pedagógicas dos docentes e da vida em geral. A comunicação como uma capacidade exclusivamente humana desde a reflexão, a construção de conhecimento e pensamento, tem permitido evidenciar o boom das tecnologias no mundo globalizado, encontrando outras formas de comunicação, de difusão e construção de saberes. Este projeto pretende fortalecer processos comunicativos que sustentem e tenham validez à formação de saberes com o uso das TICS nas práticas pedagógicas dos docentes através das conformações de redes e comunidades de aprendizagem.

\section{Palavras chave}

Comunicação docente, práticas pedagógicas, estratégias pedagógicas, atitude docente, Tecnologia da Informação e Comunicação (TIC)

\section{Introducción}

El programa "Representación ontológica hipermedial en línea para el aprendizaje significativo" se propone, a través del proyecto "Efecto del ambiente digital SIMAS en la comunicación, la actitud y las estrategias pedagógicas utilizadas por docentes: validación de dos áreas y niveles del sistema educativo", modificar y extender el software del Sistemas de Marcos para el Aprendizaje Significativo (SIMAS), para que pueda ser operado desde Internet; evaluar el efecto que tiene un ambiente de aprendizaje basado en representaciones ontológicas, hipermediales y de carácter colaborativo en los docentes que apropian esta plataforma para sus prácticas educativas; promover la conformación de una comunidad virtual que apropie el software que se desarrolla para la construcción colaborativa del aprendizaje significativo, con proyección a la educación básica, media-vocacional y superior, y contribuir con la formación de nuevos investigadores en el desarrollo de este programa.

Frente a la indagación y apropiación sobre la implementación del uso de medios tecnológicos en la producción de saber y la creación de sociedades de conocimiento, surge la pregunta de investigación: ¿̇ué efecto genera una plataforma digital basada en la construcción de representaciones ontológicas, hipermediales y colaborativas en la selección de estrategias pedagógicas utilizadas por los docentes en procesos educativos? En consecuencia, profundizar en el estudio basado en la construcción de representaciones ontológicas e hipermediales se convierte en una posibilidad de abrir otros caminos frente a la formación de maestros, quienes al abordar sus prácticas pedagógicas contribuyan al mejoramiento de la calidad de la educación, permitiendo que las tecnologías de la información y la comunicación (TIC) sirvan como medios para favorecer la gestión del conocimiento.

En este artículo se presenta el estado del arte a partir de tres categorías preestablecidas, a saber: a) prácticas y estrategias pedagógicas utilizadas por docentes mediadas por las TIC, b) actitud de docentes en ambientes de aprendizaje mediados por tecnología y c) estrategias de comunicación docente en ambientes de aprendizaje. 


\section{Prácticas y estrategias pedagógicas utilizadas por los docentes, mediadas por TIC}

La práctica pedagógica dentro del contexto educativo puede determinarse como una acción de intervención que tiene como propósito alcanzar la formación de sujetos cognoscentes a través del desarrollo de habilidades y competencias que les permitan desenvolverse en un mundo cada vez más globalizado. Estos procesos de intervención deben pensarse desde el uso de estrategias, procesos y experiencias contextualizadas; es decir, es necesario tener en cuenta los intereses, habilidades y necesidades de formaciones de niños, niñas y jóvenes que asisten a los diferentes espacios de aprendizaje.

En Colombia la educación se define como un proceso de formación permanente, personal cultural y social que se fundamenta en una concepción integral de la persona humana, de su dignidad, de sus derechos y de sus deberes (MEN, 2005a). En este sentido no se puede desconocer que una de las intencionalidades del Sistema Educativo Colombiano a través del Ministerio de Educación Nacional y en aras de la formación integral, es permitir la formación en uso de TIC para la aprehensión, comprensión y creación de conocimiento.

En relación con lo anterior, González (2008) muestra que las diferentes organizaciones de la sociedad han buscado transformar los procesos para hacerlos más eficientes, involucrando el uso de TIC, y es aquí donde los estudiantes están llamados a desempeñar un papel fundamental en este ámbito, ya que suelen hallarse a la vanguardia de la utilización de las nuevas tecnologías al incorporarlas en sus prácticas cotidianas. La Unesco (2005) afirma que las personas de más edad (profesores) también están destinados a desempeñar un papel importante, porque cuentan con la experiencia necesaria para compensar la relativa superficialidad de la comunicación en tiempo real e igualmente impedir que se olvide que el conocimiento es, en esencia, un camino hacia la sabiduría. Por tanto, esto generará una innovación necesaria e indiscutible en la actitud y las prácticas pedagógicas de los docentes. González (2008) encontró que, casi en su mayoría, los docentes cuentan con computadora personal con acceso a Internet, utilizan el correo electrónico y se están actualizando en cursos del uso de TIC; involucran en sus prácticas educativas presentaciones en diapositivas, como herramienta para las exposiciones. No obstante, es necesario adelantar con los maestros un proceso de sensibilización que permita un cambio de actitud frente a nuevas funciones y desafíos de sus prácticas educativas.

Además, cabe mencionar que la práctica pedagógica tiene sentido si en ella se expresan las intenciones conceptuales y socioculturales de los maestros, quienes se encargan de orientar los procesos de otros sujetos (niños, niñas y jóvenes). En ese sentido, la práctica pedagógica es un espacio de intersección de esas intenciones y construcciones conceptuales dinámicas socioculturales de carácter histórico, porque las prácticas se ubican en espacios históricos concretos; 
es decir, las intervenciones pedagógicas que hoy se realizan hubieran podido tener validez en los tiempos de Santander y Bolívar, pero no se podría pretender que tuvieran valor dentro de 50 o 100 años, para responder o abordar los conflictos que les retan y que les permiten construirse y decantarse (N. Rodríguez, comunicación personal, 16 de junio de 2014).

La labor del docente enmarca en gran medida el éxito del proceso educativo. Por ello son de gran importancia las estrategias pedagógicas que aquel utilice para conseguir el objetivo de aprendizaje propuesto. Dichas estrategias deben ajustarse al contexto, a las necesidades e intereses de los estudiantes, al horizonte institucional y a las exigencias de un mundo globalizado y avanzado tecnológicamente. Para definir las estrategias pedagógicas, dice Mockus (1984) que son las acciones realizadas por el maestro con el fin de posibilitar la formación y el aprendizaje de las diferentes áreas del conocimiento adelantadas por los estudiantes. Así, se ratifica la relevancia del docente en el proceso educativo, como facilitador que debe estar a la vanguardia y proporcionar al estudiante caminos atractivos, efectivos y modernos que propicien aprendizajes significativos.

Salas (2000) reflexiona sobre la posibilidad de apreciar a las TIC como una estrategia para abordar, mejorar y transformar las prácticas pedagógicas, y resalta que por este medio se puede garantizar la igualdad de condiciones en la participación y construcción de saberes, tanto de estudiantes como de maestros. Hace alusión a la educación superior en Cuba, sobre todo en las facultades de Medicina, concluyendo que las TIC son mediadoras de la construcción y acceso al conocimiento pertinente, equitativo y de calidad, no solo en el nivel superior o universitario, sino que pueden y deben vincularse a los demás niveles del sistema educativo.
Con respecto a las comunidades virtuales, como estrategias pedagógicas, Almudena y Suárez (2010) citan la siguiente definición de Rheingold: "[...] agregaciones sociales que emergen de la red cuando un número suficiente de personas entablan discusiones públicas durante un tiempo lo suficientemente largo, con suficiente sentimiento humano, para formar redes de relaciones personales en el ciberespacio" (p. 3). Este escenario facilita la integración de agentes de software para diferentes funciones pedagógicas, como por ejemplo, la generación de preguntas; la activación de juicios de metamemoria y la gestión de metas; la gestión y monitoreo de estrategias de aprendizaje, y la gestión de actividades colaborativas en línea.

Meza, Pérez y De la Barreda (2002) definen a las comunidades virtuales como una herramienta didáctica para el apoyo de la labor docente. Destacan, como estrategia para la puesta en marcha de las comunidades, el desarrollo de habilidades de autoaprendizaje en el docente; es decir, que aprenda a aprender, implicando con ello el análisis, la comprensión y el uso de la información, así como el trabajo en equipo con otros docentes, la formación comunidades que responden a intereses académicos y la generación de ambientes de aprendizaje que redundan en el desarrollo profesional del docente en el aula.

En la misma línea, Salinas (2004) indica que se están desarrollando nuevos sistemas que pretenden explotar adecuadamente las potencialidades comunicativas de las TIC, tanto en el caso de aplicaciones en tiempo real, como los sistemas asincrónicos y de hipermedia. Plantea que dichos sistemas son verdaderas posibilidades de accesibilidad, flexibilidad y costos para docentes y estudiantes. De este modo, además, expone algunas experiencias desarrolladas en proyectos e-learning, teleformación, enseñanza semipresencial, entre 
otras, aplicadas en instituciones de educación superior y empresas, que hacen evidente un sesgo, dependiendo del origen, el tiempo, la organización, etc. En su estudio destaca tres enfoques: a) el tecnológico, que parte de la idea de la sofisticación del entorno tecnológico (proceso de construcción del conocimiento y no de distribución de información); b) el de contenido, contrario al enfoque excesivamente tecnológico, que basa la calidad del proceso en los contenidos y en la representación del conocimiento que estos ofrecen, y c) el metodológico, centrado en el estudiante, que se sustenta en una combinación equilibrada entre las decisiones tecnológicas, pedagógicas y organizacionales.

La metodología que se describe en el libro Cambios metodológicos con las TIC hace alusión al constructivismo resumido en la siguiente frase: "Enseñar a pensar y actuar sobre contenidos significativos y contextuados" (Díaz y Hernández, 1999, p.13-19). Se observa la necesidad de evidenciar la capacidad y competencia del docente para diseñar y aplicar estrategias que permitan a sus estudiantes aprender significativamente.

Marchisio, Plano, Ronco y Von (2005) en su investigación "Combinación de estrategias didácticas e integración de TIC en la enseñanza de fundamentos de física cuántica para Ingenieros", adelantada en Facultad de Ciencias Exactas, Ingeniería y Agrimensura de la Universidad Nacional de Rosario (Argentina), dan cuenta de algunos avances de un proceso investigativo que busca mejorar o facilitar la enseñanza/aprendizaje de la física cuántica con la integración de las TIC.

Arias (2010) afirma: "[...] los estudiantes que interactúan con un ambiente b-learning presentan niveles más altos de motivación y uso de las estrategias de aprendizaje que los estudiantes de un ambiente presencial" (p. 149). Por su parte, Batista et al. (2008, p. 17) citando a Anthony Giddens, argumenta: "[...] está surgiendo una nueva forma de sociedad ante nuestros ojos. Y esta sociedad no es algo ajeno a nosotros. Está aquí, en nuestras vidas personales, en nuestras emociones, en las ansiedades que enfrentamos todos los días". Atendiendo estas consideraciones, la afirmación anterior recoge lo que la autora plantea a través de la propuesta en formular seis trazos en los que recoge situaciones de la vida cotidiana en Argentina (tabla 1). 
Tabla 1. Seis trazos en los que recoge situaciones de la vida cotidiana en Argentina

\begin{tabular}{|c|c|c|c|c|c|}
\hline Primer trazo & Segundo trazo & Tercer trazo & Cuarto trazo & Quinto trazo & Sexto trazo \\
\hline $\begin{array}{l}\text { "Lo importante } \\
\text { es el chip } \\
\text { mamucha" } \\
\text { (p. 20). }\end{array}$ & $\begin{array}{l}\text { "Menos de } 50.000 . \\
\text { ultrapasteurizada. } \\
\text { Fortificada con } \\
\text { calcio y vitaminas A, } \\
\text { C y D" (p. 22). }\end{array}$ & $\begin{array}{l}\text { "Argentinos en el } \\
\text { exterior" (p. 26). }\end{array}$ & $\begin{array}{l}\text { "El planeta en peligro" } \\
\text { (p. 27). }\end{array}$ & $\begin{array}{l}\text { "Live 8, } \\
\text { organizado por } \\
\text { Make poverty } \\
\text { history" (p. 29). }\end{array}$ & $\begin{array}{l}\text { "La revolución de } \\
\text { las TIC" (p. 32). }\end{array}$ \\
\hline $\begin{array}{l}\text { Se hace evidente } \\
\text { el alejamiento } \\
\text { generacional } \\
\text { entre niños } \\
\text { y adultos } \\
\text { (niños, padres, } \\
\text { docentes, } \\
\text { abuelos). }\end{array}$ & $\begin{array}{l}\text { Menciona la } \\
\text { influencia de las } \\
\text { TIC en la sociedad } \\
\text { de consumo y } \\
\text { las ventajas que } \\
\text { tienen para la } \\
\text { comercialización de } \\
\text { diversos productos. }\end{array}$ & $\begin{array}{l}\text { Ubica a las TIC } \\
\text { como un medio de } \\
\text { acercamiento que es } \\
\text { usado por argentinos } \\
\text { que se encuentran } \\
\text { fuera del país, para } \\
\text { permanecer en } \\
\text { contacto con sus } \\
\text { familias. }\end{array}$ & $\begin{array}{l}\text { Cita a Morin quien } \\
\text { plantea que "se } \\
\text { necesita reforma } \\
\text { del pensamiento, } \\
\text { que permita unir, } \\
\text { contextualizar, } \\
\text { globalizar y, al mismo } \\
\text { tiempo, reconocer lo } \\
\text { singular, lo individual, } \\
\text { lo concreto" (p. 28). }\end{array}$ & $\begin{array}{l}\text { Habla de la } \\
\text { desigualdad } \\
\text { tanto } \\
\text { económica, } \\
\text { como de } \\
\text { accesibilidad al } \\
\text { uso de recursos } \\
\text { tecnológicos } \\
\text { entre países. }\end{array}$ & $\begin{array}{l}\text { Destaca la } \\
\text { importancia de } \\
\text { lo que se } \\
\text { considera la } \\
\text { revolución en las } \\
\text { tecnologías de la } \\
\text { información y la } \\
\text { comunicación. }\end{array}$ \\
\hline
\end{tabular}

Fuente: adaptación de Batista (2007)

Los trazos que describe la autora hacen alusión a situaciones cotidianas en las que se usan las TIC, pero sobre todo demarcan el camino que debieran seguir estas tecnologías para disminuir la tasa de pobreza, para promover la equidad, el conocimiento, generar estrategias, cuidar el planeta, entre otros.

Lo que resulta un potencial indudable para la tarea pedagógica es la posibilidad que brindan las nuevas tecnologías de democratizar la producción y convertir sus herramientas en instrumentos de autor. La posibilidad de personalizar estos recursos dependerá de los contextos de apropiación significativa que cada institución escolar pueda construir entre todos los agentes y sujetos que participen en sus proyectos de integración. (Batista, 2007, p. 33)

La misma autora plantea tres claves para integrar las TIC a la escuela: a) construir relación con las tecnologías (participar en comunidades virtuales, automatizar tareas); b) producir contenidos y publicarlos en la Web; c) considerar el volumen de la información (leer, seleccionar, evaluar, cuestionar) y concebir otras formas de organización de la informa- ción (audiovisual, multimedia, hipermedia). Estas tres claves las asume como directrices que permiten encontrar oportunidades para desarrollar habilidades a través de las TIC que tienen que ver con "la distribución ingeniosa del pensamiento y aprendizaje" (Batista, 2007, p. 38). Finalmente, plantea que las oportunidades antes mencionadas hacen de las TIC una suma de herramientas significativas, entre las que destacan los mapas conceptuales digitales, Webquest y Weblogs.

Maldonado et al. (2008) describen la experiencia en la conformación de redes de aprendizaje en el colegio INEM de Bucaramanga y dos colegios de Bogotá. El proyecto orientó su desarrollo en la resolución de dos interrogantes:

- ¿ ¿Cuál es el efecto de incorporar los escenarios SIMAS y Cool Modes en el desarrollo de competencias básicas en matemáticas, ciencias naturales, lenguaje y vida ciudadana, medidas a través de evaluación de logros y pruebas estandarizadas?

- $\quad$ ¿Cuál es el efecto de un modelo dinámico de comunicación, apoyado por 
el Portal Colombia Aprende, en la consolidación de una comunidad de aprendizaje visualizada a través de la evolución en negociación de metas y estrategias pedagógicas, frecuencia y eficacia de la comunicación, y permanencia de los participantes? (p. 2).

La puesta en marcha del proyecto antes referenciado requirió de la conformación de tres comunidades de aprendizaje donde participaron investigadores (expertos y semilleros), docentes de cuatro asignaturas diferentes en los tres colegios y estudiantes. Comunidades que interactuaron a través del software de representación de conocimiento mediante ontologías (SIMAS), un software para la representación gráfica y solución colaborativa de problemas (Cool Modes) y una plataforma web para fomentar la construcción de la comunidad (Portal Colombia Aprende). En cuanto a los resultados del anterior estudio, dicen los autores que la investigación no arrojó los efectos deseados (vínculos de la red con los actores involucrados). Hallaron algunas dificultades que obstaculizaron el trabajo de planeación colaborativa, tales como: la poca intervención de los docentes, debido a la baja comunicación que se estableció en la red; problemas en las condiciones tecnológicas; congestión en el Portal Colombia Aprende, lo que ocasionó desmotivación por parte de los estudiantes; la diferencia en los horarios de los participantes; y, la falta de cobertura de Internet.

Vergara y Castillo (2008) presentan el resultado de la investigación "SIMAS y Cool Modes en el desarrollo de competencias básicas", adelantada con estudiantes de grado décimo durante el segundo semestre de 2006 y grado undécimo en el primer semestre de 2007, pertenecientes a colegios oficiales de Bogotá, Cundinamarca (rural) y Bucaramanga. El objetivo es contribuir con la resolución de dificultades conceptuales en el aprendizaje de la física en estudiantes de secundaria y desarrollar competencias básicas relacionadas con procesos de aprendizaje, como: seleccionar, organizar, elaborar, aplicar y evaluar. La metodología consistió en incorporar con métodos colaborativos los ambientes digitales SIMAS, dispositivo didáctico que representa hipermedialmente categorías y relaciones básicas desde ontologías y Cool Modes, dispositivo digital para la solución de problemas con ayuda de simuladores. Utilizaron el Portal Colombia Aprende para la interacción entre los participantes de los colegios, conformando así una comunidad de aprendizaje. Plantearon como resultados, la potencialización de una comunidad de aprendizaje, bajo el concepto "Nosotros"; el trabajo colaborativo de quienes se involucraron en la investigación apoyados por los ambientes SIMAS y Cool Modes, habilitando el desarrollo de competencias cognitivas, la metacognición individual, la metacognición social y la consolidación de comunidad. Con la ayuda de estos ambientes, la mayoría de los estudiantes afianzaron y categorizaron los conceptos trabajados, aplicándolos a la solución de situaciones problémicas. Además, los estudiantes demostraron ser competentes 
en la búsqueda, selección y organización de la información y establecieron las interrelaciones existentes entre las variables que describen los fenómenos físicos estudiados.

Bosco (2008) advierte sobre la necesidad de desarrollar determinadas competencias y las perspectivas metodológicas aconsejables para la formación. Plantea el uso de las TIC desde tres dimensiones que deben ser conocidas y reconocidas por los docentes: a) la dimensión instrumental, se refiere al uso de los instrumentos o recursos tecnológicos; b) la dimensión cognitiva, hace alusión al desarrollo de habilidades para buscar, seleccionar e integrar información, y c) la dimensión actitudinal, que tiene que ver con el desarrollo de valores y actitudes hacia la tecnología. Concluye diciendo que es urgente que los docentes conozcan y transmitan no solo un uso práctico (dimensión instrumental), sino también un uso crítico (dimensión cognitiva) y ético (dimensión actitudinal), en el uso de las TIC.

Según Zappala, Köppel y Suchodolski (2010), "la utilización de distintos formatos digitales (imagen, audio, texto y video) estarán presentes en distintas propuestas permitiendo el desarrollo de actividades y la producción de materiales en distintas formas de lenguaje (oral, escrito, gestual)" (p. 4); además, los mismos autores consideran que la oportunidad que ofrecen los medios y recursos digitales, hace posible el planteamiento de estrategias y actividades que respondan a las necesidades e intereses de los estudiantes. En su trabajo presentan una serie de propuestas que permiten la construcción de conocimiento a partir de la producción de videos (por estudiantes y docentes), entre las que destacan: a) el aprendizaje visual desde organizadores gráficos para presentar la información; b) aplicaciones digitales como Cronos, CMap-Tools que organizan la información; c) búsqueda de información en Internet; d) la Web 2.0 que promueve la colaboración e interacción entre los usuarios. Y concluyen: "[...] las herramientas y recursos digitales, no configuran por sí mismos un contexto de aprendizaje, dependen siempre de la intencionalidad docente con base en los objetivos, contenidos y competencias que desee desarrollar en el trabajo con sus estudiantes" (p. 10).

El uso de las tecnologías en la escuela debe facilitar el desarrollo de medios alternativos, de producción de conocimientos y de pensamiento; es decir que, al hacer uso de las herramientas tecnológicas y digitales, el docente debe preguntarse el impacto de estas en las prácticas pedagógicas, la significación de esta frente al proceso de producción, el papel del saber, la comunicación y la interacción con otros a través de redes. Además, las tecnologías de la información y la comunicación son necesarias para la propia formación y desenvolvimiento diario de docentes y estudiantes; repercute positivamente en la significatividad y calidad de la enseñanza-aprendizaje; aporta, entre otros beneficios, flexibilización de la enseñanza, el aprendizaje cooperativo, la enseñanza individualizada y el autoaprendizaje y, por consiguiente, el desarrollo integral de la personalidad de los educadores.

\section{Actitud de docentes en ambientes de aprendizaje mediados por tecnología}

El docente es un comunicador de actitudes y, más aún, cuando posee características o conocimientos que el alumno no tiene y desea alcanzar. Curtis (1962) afirma que las actitudes están en relación con la predisposición de sentir, percibir, pensar y comportarse ante un objeto. Según este autor existe diferencia entre comportarse, sentir o pensar; es así que un docente puede hacer uso de las TIC sin que 
le signifiquen un aporte importante a la educación o sentirse inseguro al utilizarlas en clase. Por tanto, se toma la actitud como una organización psicológica particular, o sea, con una entidad propia.

López (2009) cita a González (1981) para presentar tres componentes, que servirán como organizadores de la actitud docente: a) cognoscitivo o perceptivo, formado por percepciones y creencias hacia un objeto, o por la información que se tiene de él; si no se conoce un objeto no se puede generar una actitud hacia él; b) afectivo o sentimiento más característico de las actitudes, permite estar en favor o en contra de un objeto social relacionado con creencias y opiniones, y c) conativo o de conducta, componente activo de la actitud, que consiste en la tendencia a reaccionar hacia los objetos de una determinada manera. El componente conativo, establece una relación entre actitud/conducta y las variables que intervienen en el quehacer docente. Para empezar a relacionar el proceso de enseñanza con la actitud que tiene el docente frente al uso de TIC en su desempeño profesional, se reconocen, además, tres áreas del desarrollo humano: conceptual, operativa y afectiva; las dos primeras son manejables, pero la última es donde se presenta la problemática, que en palabras de Verdugo $(2004$, p. 3) significa que "hace más el que quiere, que el que puede".

Existen variadas posturas que hacen referencia a la actitud negativa hacia la incorporación de las TIC en sus clases por parte del docente. Tres de las más significativas son: a) el fracaso de introducir TIC en el aula, atribuible a la falta de habilidad de los docentes para adaptar sus estilos de enseñanzas con las tecnologías, los maestros pueden sentirse amenazados por el cambio y por ello se resisten a ello (Hannafin y Savenye, 1993); b) frustración experimentada en el proceso de aprendizaje del uso de las TIC (Sandholtz, Ringstaff y Dwyer, 1990), y c) algunos maestros simplemente no creen que las TIC puedan mejorar los resultados de aprendizaje, por miedo a perder el control escénico o por temor a sentirse tontos frente a sus estudiantes (Wiske, 1990). Así, para los anteriores autores, la transformación que producen las innovaciones tecnológicas en educación debería ser introducida por un cambio voluntario de los docentes; no solo por la propaganda de expertos o por exigencias de decretos gubernamentales.

Martín, Lucena y Díaz (2002) presentan en su investigación la actitud de docentes y futuros docentes respecto a la formación de las TIC aplicadas a la educación, con participantes elegidos aleatoriamente de diferentes instituciones educativas, más concretamente de la Universidad de Granada. Las actitudes fueron evaluadas con una escala de Likert, teniendo en cuenta las siguientes dimensiones: a) aplicabilidad de las TIC en primaria; b) importancia de la formación en TIC, y c) formación inicial en TIC aplicadas a la información y la formación permanente en TIC aplicadas a la educación. Encontraron que las actitudes de los docentes eran bastante positivas respecto al uso de las TIC en el aula y reconocieron la importancia de la formación para su uso didáctico; la mayoría de los docentes consideraron que podían utilizarlas en el desarrollo de las áreas 
del currículo, en unas más que en otras; la gran mayoría demostraron confianza en ellas como un medio didáctico; consideraron que es esencial la formación en TIC aprovechando los beneficios y ventajas que tiene su uso, al reconocer que mejora el desarrollo profesional. Con respecto a la formación inicial, los investigadores concluyeron que es de baja calidad y esto hace que falte o se necesite mayor capacitación para afianzar la utilización de estos medios. En cuanto a la formación permanente, se evidenciaron costos elevados o ausencia de ofertas, además de ser demasiado instrumentalistas y el tiempo que se dispone para dicha capacitación es muy escaso, por lo cual, conciben que es indispensable contar con una actitud más positiva, activa y definitiva, y tener siempre una intencionalidad clara y una gran motivación para el aprendizaje.

Cervera (2002) realiza un razonamiento en torno a su experiencia y, desde una revisión bibliográfica, argumenta:

Los cambios y redefiniciones que se han producido en el mundo educativo a partir de la incorporación masiva de las TIC, así como del impacto de estos cambios en la formación inicial y permanente del profesorado. Enfatiza en la necesidad de considerar no solo los espacios educativos formales, sino también los no formales y los informales. Describe los roles de un profesor en entornos tecnológicos, puntualiza los cinco ámbitos (redes, materiales, periféricos, ofimática y estrategias de comunicación) que considera fundamentales para la formación del profesorado en TIC y plantea sugerencias en cuanto al proceso de formación potenciando la noción de autoaprendizaje. Termina asegurando que el maestro hoy, para el cabal desempeño de su rol, debe ser un usuario experimentado en TIC (p. 49).
Cañuta (2005), en su trabajo de maestría, describe las actitudes de los docentes frente al uso de las TIC y su incidencia con los estilos de aprendizaje, basados específicamente en el modelo de programación neurolingüística (PNL). A través de un estudio descriptivo de ambas variables, afirma que es posible conocer el grado de actitud favorable o desfavorable de los docentes frente al uso de las TIC cuando las utilizan como apoyo para el aprendizaje de alumnos. Además, determina los estilos de aprendizajes predominantes en los docentes y su incidencia en sus actitudes, proporcionando nuevos antecedentes sobre su grado de aceptación hacia las concepciones del proceso de enseñanza y aprendizaje. Las variables fueron medidas a través de los resultados obtenidos en la aplicación de la escala de actitud docente hacia las nuevas tecnologías y el inventario sobre estilos de aprendizaje aplicados a 75 docentes. A partir de los resultados obtenidos se destaca la actitud favorable que presentaron los docentes hacia el uso de las TIC y su incorporación en los procesos educativos de enseñanza/aprendizaje. Los docentes presentaron un dominio más alto en el sistema de representación visual. Como recomendación propone una mirada crítica hacia las TIC, por considerar que posibilita ampliar los conocimientos, adquirir mayor información y garantizar el mejoramiento de la calidad educativa y de las prácticas pedagógicas.

Zapata (2006) afirma que en la actitud de los docentes ante el diseño instruccional tecnológico se evidenciaba indiferencia ante los objetos de aprendizaje, por el temor de enfrentarse a los diseños e innovaciones tecnológicas que aportarían a sus prácticas pedagógicas. El autor propone un diseño instruccional accesible con unos principios fundamentales como: diferenciar el contenido de la estructura y de la presentación; permitir la 
personalización de las aplicaciones informáticas de acuerdo con las preferencias y necesidades de los estudiantes; garantizar el acceso integral y la interacción con teclados de fácil uso; además, proporcionar suficiente información acerca del contexto y orientación para la navegación de manera que se pueda crear una herramienta que permita a los docentes alcanzar estos objetivos.

En la investigación adelantada por Álvarez et al. (2011) se presentan las actitudes de los docentes de la Universidad de Valladolid, en la que se analiza que la presencialidad en el aula ha disminuido debido a que se han introducido nuevos canales de comunicación entre el docente y el estudiante, así como modelos de aprendizajes colaborativos con el uso de TIC. En esto ha influido el proceso de adaptación y convergencia en el que están inmersos los actores. Como conclusión de la investigación se identifica que la integración del mundo de las TIC depende en gran medida de los maestros y por esto son fundamentales los conocimientos, percepciones y actitudes de los maestros, motivando en ellos la actualización permanente para potenciar sus competencias y destrezas con estos ambientes de aprendizaje.

En un trabajo etnográfico realizado durante dos años por Moreira (2005) con el objetivo de analizar el proceso de la integración de uso de TIC en las prácticas de enseñanza aprendizaje en la básica y media de Canarias, el autor concluyó que las TIC propician algunos cambios significativos en la organización de las instituciones de educación, y su utilización, por parte de los docentes, es más baja en primaria que en secundaria. Afirma, además, que es claro que los maestros introducen con diferentes grados de aplicación, profundidad y calidad las TIC, por tanto no se convierte en un replanteamiento significativo de los modelos de los docentes y de los aprendizajes. Por su parte, Vence (2011) argumenta que los profesores deben crear entornos de aprendizaje interactivos con el propósito de mejorar sus prácticas durante el proceso de enseñanza/ aprendizaje, estimulando a partir de las TIC, los procesos mentales haciéndolos a su vez, más significativos.

Valdés et al. (2011) presentan un estudio cuantitativo transeccional llevado a cabo en instituciones educativas de México, como análisis de resultados de evaluaciones de docentes sobre el uso de las TIC y su implicación en la calidad educativa. El propósito es describir las actitudes de los docentes hacia las TIC y su relación con variables sociodemográficas (edad y género) y con aquellas que indican posibilidades de acceso a las tecnologías (computadora en el hogar y enciclopedia en el salón de clases). En los resultados se señala que, de manera general, los docentes poseen actitudes positivas hacia las TIC, especialmente los de género masculino, y aquellos que cuentan con mayores posibilidades de interacción tecnológica. A pesar de que las actitudes de los maestros son positivas, son menos favorables ante los factores de factibilidad y disponibilidad por la deficiencia de los programas de capacitación y mejor disposición de aquellos docentes que cuentan con mayores posibilidades de interacción. Es decir, la 
actitud de los maestros frente a las TIC es un factor fundamental para determinar su uso en las prácticas pedagógicas.

Martínez (2013) presenta un informe de investigación basado en un estudio de caso sobre la actitud pedagógica del profesor Filex (programa de maestros en TIC) de la Universidad de Guadalajara. Desde un marco constructivista sociocultural muestra el tema de la integración tecnológica en la enseñanza del inglés focalizado desde la experiencia y actitud del docente Filex hacia las TIC. La problemática que origina la investigación es la forma desigual y lenta como se han venido integrando las TIC en la formación docente, incorporadas con mayor facilidad en el ámbito escolar administrativo que en el de la formación y práctica docente. En el estudio recurre a diversos autores para concluir que: a) a mayor tolerancia a la frustración, expresada por docentes en el uso de herramientas TIC, mayor es el acercamiento a dichas herramientas. b) Es importante desarrollar la capacidad de autoobservación para desvirtuar el temor a perder el control de la clase cuando se utilizan herramientas TIC. c) Se evidencia que los docentes reconocen y se identifican con el enfoque constructivista de la educación pero las observaciones de su práctica en el aula reveló que no utilizan las TIC como instrumentos para promover la resolución de problemas, el aprendizaje colaborativo o la autorregulación del aprendizaje.

Patiño y Sallé (2014), desde un enfoque biográfico narrativo -una mirada crítica a la actitud de los maestros en cuanto a la apropiación de TIC en sus prácticas pedagógicas-, encuentran diversas actitudes como resistencia, inclusión, aceptación e implementación de tecnologías. Destacan la importancia de incluir en el proceso formativo el uso de las TIC a los niños y a los padres de familia para reducir la brecha digital a partir de los altos niveles de accesibilidad a Internet y el alto impacto que tiene esta realidad en las prácticas de los docentes.

Existen estudios que presentan la actitud del docente como un estado que afecta lo conceptual, lo anímico, lo ético como ser humano en el proceso educativo; pero no existe claridad para modular, reflexionar y perfeccionar la actitud y apostarle al mejoramiento de la calidad de la educación. Es importante mostrarle al maestro que través de la tecnología, puede transformar positivamente la actitud en el aula, en calidad de sujetos activos (docentes, estudiantes, padres, etc.) que construyen y reconstruyen con recursos tecnológicos, potenciando así la necesidad o disposición para aprender.

\section{Estrategias de comunicación docente en ambientes de aprendizaje}

El concepto de comunicación ha mantenido su esencia a través de la historia su esencia; debido a que es un proceso y un medio. En la medida que ha ido evolucionando el ser humano, creciendo, aprendiendo, tecnificando, construyendo, revolucionando su existencia con el uso de las TIC y en pro de ella misma, lo ha hecho también en la escuela, como reflejo de la sociedad. De esta manera, ha evidenciado que la comunicación y la interacción son elementos esenciales para la construcción de redes de conocimiento, como canales inmersos hoy por hoy en el mundo digital, y ha incorporado las nuevas tecnologías en los procesos de enseñanza aprendizaje dando lugar a la llamada sociedad de la información, en la que todo y todos están comunicados en un proceso de globalización que traspasa fronteras y une países a nivel económico, social, cultural y en otros ámbitos vitales de la existencia humana. 
Según Fonseca (2000, p. 4) la comunicación es una cualidad racional y emocional de los seres humanos que surge de la necesidad de ponerse en contacto con los demás, intercambiar información, transmitir ideas o intercambiar mensajes o significados que adquieren sentido de acuerdo a intereses y experiencias previas compartidas, mediante un conjunto común de símbolos, constituyéndose así en uno de los procesos fundamentales de la experiencia humana y la organización social, como lo afirma Chiavenato (2006).

Sainz (1998) destaca la importancia de la comunicación en la formación del ser humano e identifica las tres principales dificultades presentes en la comunicación pedagógica: el estilo de comunicación autoritario y verticalizado, que sigue prevaleciendo en las instituciones educativas; el estilo de comunicación autoritario centrado en la información que prevalece en la relación profesor/ alumno o comunicación pedagógica autoritaria; $y$, la dificultad en la interacción comunicativa grupal. Explica que la importancia de desarrollar procesos pedagógicos para favorecer y propiciar el mejoramiento de procesos comunicativos entre los miembros de la comunidad educativa, aprovechando la vigencia y prevalencia que tienen el uso de las TIC en la sociedad actual, que facilitan formas de relaciones generando mejoras en la comunicación de las IE, constituye un elemento fundamental de las prácticas pedagógicas de los maestros. Como conclusión asegura que la comunicación es un proceso determinante para el desarrollo de la subjetividad humana, que expresa la interacción entre los sujetos de la actividad y permite la apropiación sociohistórica, así como la construcción y producción del conocimiento y hace necesaria su democratización a través de estilos de dirección y comunicación más abiertos, flexibles y participativos, lo que facilita una mayor autonomía y responsabilidad de los sujetos que intervienen en este proceso.

Por su parte, Meira (2001) relaciona el campo de la comunicación con las nuevas tecnologías y la educación ambiental. Afirma que ambas disciplinas han abierto posibilidades para abordar problemáticas que aquejan al ser humano en diversos campos del saber. Relaciona las NTIC con la educación ambiental, afirmando que aporta a los procesos de comunicación en la construcción de conocimiento, en la medida que permite la rápida difusión, comunicación y visibilización de problemáticas y transformaciones sociales posibilitadas desde la acción pedagógica. El objetivo de esta investigación es realizar una reflexión sobre los cambios y aplicaciones que pueden introducir las NTIC en la investigación relacionada con la educación ambiental estimando el progreso continuo en íntima alianza de la informática y las telecomunicaciones. Esto se constituye como el eje tecnológico vertebrador de la nueva sociedad con una serie de herramientas, sistemas tecnológicos y programas que reúnen cualidades esenciales, además de permitir gestionar y transferir, mejor y en menor tiempo, grandes cantidades de información, preferentemente digitalizada, que se codifica y se presenta en distintos soportes y lenguajes (sonido, texto e hipertexto, imagen fija o móvil, multimedia e hipermedia, etc.) facilitando nuevas formas o 
entornos para una comunicación interactiva y simultánea. Muchos de estos conocimientos y métodos que exploran las posibilidades pedagógicas de las NTIC se han establecido gracias a derivaciones y aplicaciones concretas dentro de la investigación educativa.

Es preciso recoger el aporte teórico que Hopenhayn (2002) hace a la comunicación y las prácticas pedagógicas cuando afirma que la transformación y reformas educativas en Latinoamérica se desarrollan desde la innovación y la capacidad de construir ciudadanía en contextos democráticos y generación de redes de conocimiento, haciendo necesario educar en la sociedad de la información con equidad; la calidad se constituye en una respuesta a la inequidad y desigualdad en el acceso y manejo de las redes de información y medios audiovisuales para que la producción de conocimiento circule, se comunique y se renueve constantemente; en consecuencia, las TIC se convierten en la posibilidad de dialogar con el resto del mundo desde una posición globalizada de todos y para todos, cuando realmente se garantiza el acceso a ellas.

En la década anterior se adelantaron estudios acerca del uso de las TIC y sus consecuencias, los cuales visualizaron el aporte que podría tener en los procesos comunicativos, y que hoy, diez años después, siguen siendo pretensiones a las que se apunta desde la formación. Berríos y Buxarrais (2005) ofrecen un estudio de la relación que los adolescentes establecen con las TIC. Estas tecnologías expanden las posibilidades de la comunicación, generan nuevas culturas y posibilitan el desarrollo de nuevas habilidades y formas de construcción de conocimiento. Para esto es necesario considerar que las TIC otorgan múltiples oportunidades y beneficios; favorecen las relaciones sociales, el aprendizaje cooperativo, desarrollo de nuevas habilidades, nuevas formas de construcción del conocimiento, y el desarrollo de las capacidades de creatividad, comunicación y razonamiento (Castells y Macías, 2002, p. 24).

Tessio, Rumbado y Porro (2006) describen y justifican en su investigación, la importancia del diseño de un programa de formación de docentes en TIC como hiperlectores y formadores de hiperlectores, que trascienda la formación mecánica; incorpore y promueva la reflexión sobre su uso social en el proceso educativo; desarrolle el pensamiento crítico y propicie el mejoramiento de los procesos comunicativos y, por ende, educativos. Aseguran como indispensable el acceso de maestros a una alfabetización digital pertinente y a tono con la globalización, para logar una relación más flexible y dinámica con los estudiantes, propiciar espacios acertados para construir conocimiento y mejorar la comunicación, a través del uso de las TIC como herramienta fundamental en las prácticas pedagógicas de los maestros.

Batista (2007) realiza un estudio en Argentina en el que reconoce la actualidad de la comunicación tecnológica. Afirma que aunque siempre ha estado presente, hoy supera históricamente otros medios de comunicación como el televisor o la radio, pues son muy diferentes los tiempos en los que se envían y reciben mensajes con respecto a la lentitud de las prácticas sociales. La escuela debe ser la responsable de apropiar los recursos tecnológicos mediante proyectos participativos, colaborativos y de integración.

También, Guitert, Romeu y Pérez (2007) plantean en su investigación que el Espacio Europeo de Educación Superior (EEES) manifiesta la importancia de definir una serie de competencias consideradas claves en la sociedad red. En este sentido, el proyecto Tuning propone un listado de las competencias que debe adquirir un estudiante a lo largo de una 
titulación universitaria, y las dividen en dos grandes bloques: a) genéricas, comunes a todas las titulaciones, como lo es el trabajo en equipo, y b) específicas, en función de la titulación. Algunas de las competencias genéricas están vinculadas al uso de las TIC. El objetivo del trabajo se centra en analizar la adquisición de las competencias genéricas en TIC a partir del desarrollo de un proyecto virtual colaborativo asíncrono en un contexto concreto: la asignatura Competencias de Trabajo en Entornos Virtuales, de la Universidad Oberta de Cataluña. Desde una aproximación al planteamiento pedagógico y a las valoraciones de los estudiantes y grupos implicados, la propuesta didáctica planteada facilita la adquisición gradual de las competencias genéricas en TIC y las propias del trabajo en equipo en un entorno virtual, dándole una gran importancia y un lugar privilegiado a las competencias comunicativas y al trabajo colaborativo.

Coll (2008) hace un análisis sobre la nueva sociedad digital y del conocimiento que ha convertido a las TIC en un eje vertebrador de la vida escolar, tanto así que los docentes las han integrado a su actividad profesional y, progresivamente, al proceso de enseñanza aprendizaje, lo cual exige de ellos mismos un aprendizaje y vivencia de una nueva cultura del aprendizaje desde tres rasgos básicos: a) los estudiantes no necesitan solamente información, sino herramientas para sistematizarla y darle sentido; b) deben desarrollar capacidades de gestión del aprendizaje, de conocimiento y de la formación en este mundo globalizado, y c) deben desarrollar competencias comunicativas que los conviertan en seres hábiles para relacionar, clasificar y sistematizar la información, para poder darla a conocer y utilizarla con pertinencia.

Arancibia, Soto y Contreras (2010), en su investigación con maestros de historia, concluyen que la escuela de la sociedad de la información no debe limitarse a ser una mera trasmisora de conocimientos; debe, además, compensar las desigualdades, fomentar el espíritu crítico, la capacidad para procesar y estructurar las informaciones, para mejorar los procesos básicos de desarrollo de los seres humanos como la comunicación, la imaginación y la inventiva.

Salmerón, Rodríguez y Gutiérrez (2010) atribuyen a las TIC los principales avances educativos y el auge en los modelos de formación, planteando que la comunicación es un elemento esencial en los procesos de enseñanza aprendizaje. Las TIC, entendidas como artefactos tecnológicos de producción cultural, facilitan la innovación desde el uso de los entornos virtuales de aprendizaje, promueven el trabajo colaborativo mediado por el computador, favorecen la comunicación, la mediación y la construcción compartida del conocimiento y superan las fronteras del aula.

Coll, Ornubia y Mouri (2007) plantean que las TIC transforman y mejoran las prácticas pedagógicas cuando junto con la comunicación se convierten en un instrumento medidor y contribuye a la relación entre estudiante y maestro dentro del proceso enseñanza aprendizaje. Coll (2004) ya reconocía que la 
desarrollo de las TIC convirtiéndose en objeto de reflexión y de investigación. Prieto (citado por MEN, 2005b, s.p.), directora de Calidad Educativa de Preescolar, Básica y Media del Ministerio de Educación afirma:

Estamos en una sociedad mediática donde nos encontramos con unas nuevas formas de comunicación y con un mayor número de mensajes. En ese sentido, todo el sistema educativo debe orientarse hacia una mejor comprensión de estos lenguajes y hacia la incorporación de estos medios en el aula de clase aportando al desarrollo de competencias comunicativas asumiéndolas como un reto pedagógico obligando al maestro a cualificarse, relacionarse y aprender a comunicar y a comunicarse con las TIC. Para ello, se encuentra importante el planteamiento del Ministerio de Educación Nacional en el apartado "la comunicación en la labor educativa", que presenta siete capacidades que deben ser desarrolladas por el educador en este proceso: comunicarse con el lenguaje oral y escrito, relacionarse e interactuar, promover y acompañar el aprendizaje, coordinar grupos de aprendizaje, usar medios audiovisuales y electrónicos, lograr una constante interlocución y producir intelectualmente en función de las necesidades del aprendizaje de sus estudiantes.

Para finalizar, se puede afirmar que hoy en día los niños y jóvenes conocen, manejan y utilizan los medios electrónicos cotidianamente. Para Feixa (2005) esta generación es la primera que ha sido educada en la sociedad digital, y Tubella (2008) la muestra como una forma de ser, de relacionarse, de compartir, cooperar, crear y difundir. Aun así, según Berríos y Buxarrais (2005) las primeras teorías de la comunicación basadas en la unidireccionalidad del mensaje y la pasividad del receptor, aún están vigentes en muchas prácticas docentes.

Según la exploración bibliográfica, es recurrente el llamado a los docentes para que fortalezcan el proceso comunicativo; esto garantiza más y mejores prácticas pedagógicas que integran las exigencias del mundo contemporáneo y las TIC pueden ser concebidas como un medio para potenciar la producción oral y escrita, al permitir compartir experiencias y presentar propuestas generadoras de conocimiento, en las que el maestro promueve y acompaña el aprendizaje, siendo un orientador dispuesto a ejercitarse con sus estudiantes, manteniendo una interlocución constante, escuchando, observando y respondiendo a sus necesidades, planeando actividades pedagógicas que hagan uso de medios audiovisuales y electrónicos con intención pedagógica.

\section{Conclusiones}

La tecnología es como esa ola, que por su gran esplendor, encanta, reta, maravilla a los estudiantes. Es reto para estudiantes y maestros es sinónimo de angustia, temor ante lo desconocido, ante lo que puede arrasar; por eso es apremiante la actualización y cualificación en TIC a los docentes para buscar y encontrar estrategias pedagógicas en ambientes digitales que permitan construir conocimientos y plantear transformaciones del mundo.

Las TIC en las prácticas pedagógicas son un recurso para acercar al desarrollo de las competencias de los estudiantes a las dinámicas del mundo contemporáneo. La Revolución Educativa propone mejorar los aprendizajes fomentando el manejo de los medios electrónicos, la televisión, la radio, el cine, el video y el impreso en el aula de clase. Los maestros 
son los ejes que movilizan el proceso para el tránsito de la enseñanza al aprendizaje aprovechando y potencializando los diversos canales de percepción del ser humano (auditivo, visual, kinestésico, entre otros).

Hoy, en el ámbito educativo, las TIC abren un abanico de nuevas posibilidades que rompen con esquemas enraizados y develan nuevas vías pedagógicas sustentadas teóricamente en la participación totalmente activa de los estudiantes, lo que enriquece de manera notable el proceso de enseñanza-aprendizaje. Se considera este como un elemento fundamental del proceso y el objetivo que SIMAS pretende alcanzar, aplicativo a través del cual los maestros pueden transformar sus prácticas pedagógicas.

\section{Referencias bibliográficas}

Álvarez, S.; Cuéllar, C.; López, B.; Adrada, C.; Anguiano, R.; Bueno, A. y Gómez, S. (2011). Actitudes de los profesores ante la integración de las TIC en la práctica docente. Estudio de un grupo de la Universidad de Valladolid. Revista Electrónica de Tecnología Educativa. [Revista en línea], 35.

Almudena, M. y Suárez, C. (2010). Las comunidades virtuales como nuevas formas de relación social: elementos para el análisis. Revista de Estudios Literarios, 43.

Arancibia, M.; Soto, C. y Contreras, P. (2010). Concepciones del profesor sobre el uso educativo de las tecnologías de la información y la comunicación (TIC) asociadas a procesos de enseñanza-aprendizaje en el aula escolar. Estudios Pedagógicos, 36(1), 23-51.

Arias, O. M. (2010). Ambientes de aprendizaje b-learning y su incidencia en la motivación y estrategias de aprendizaje en estudiantes de secundaria. Tecné, Episteme y Didaxis, 27, 130-153.

Batista, M. A. (2007). Tecnologías de la información y la comunicación en la escuela: trazos, claves y oportunidades para su integración pedagógica. Buenos Aires: Ministerio de Educación, Ciencia y Tecnología de la Nación.

Berríos, L. y Buxarrais, M. R. (2005). Las tecnologías de la información y la comunicación (TIC) y los adolescentes. OEl. Monografías virtuales: ciudadanía, democracia y valores en sociedades plurales, 5 .

Bosco, A. (2008). Las tecnologías de la información y la comunicación en la formación del profesorado: lineamientos, actualidad y prospectiva. Razón y Palabra, 13 (63). Recuperado de: http://www.redalyc.org/articulo.oa? id= 199520798002.

Cañuta, J. (2005). Actitud de los docentes frente al uso de las nuevas tecnologías de la información y comunicación y sus estilos de aprendizaje predominantes. Tesis de grado. Universidad Valparaiso, Chile. 
Castells, P y Macías, J. (2002). Un sistema de presentación dinámica en entornos web para representaciones personalizadas del conocimiento. Revista Iberoamericana de Inteligencia Artificial 16(2), 25-34.

Castillo, S. (2008). Propuesta pedagógica basada en el constructivismo para el uso óptimo de las TIC en la enseñanza y el aprendizaje de la matemática. Revista Latinoamericana de Investigación en Matemática Educativa, 11 (2), 171-194. Recuperado de: http:// www.scielo.org.mx/scielo.php?script=sci_arttext\&pid =S1 665-24362008000200002\& lng $=$ es\&tlng $=$ es

Cervera, M. G. (2002). El nuevo rol del profesor en entornos tecnológicos. 11 (1) 48-59.

Coll, C. (2004). Psicología de la educación y prácticas educativas mediadas por las tecnologías de la información y la comunicación. Recuperado de: http://virtualeduca.org/ifd/ pdf/cesar-coll-separata.pdf.

Coll, C. (ed.). (2008). Psicología de la educación virtual: aprender y enseñar con las tecnologías de la información y la comunicación. Madrid: Morata.

Coll, C.; Ornubia, O. y Mouri, T. (2007). Tecnología y prácticas pedagógicas: las TIC como instrumentos de mediación de la actividad conjunta de profesores y estudiantes. Anuario de Psicología, 38(3), 377-400.

Curtis, J.H. (1962). Psicología social. Barcelona: Grijalbo.

Chiavenato, I. (2006). Introducción a la teoría general de la administración. 7a. ed. Madrid: McGraw-Hill Interamericana.

Díaz, B. F. y Hernández R. G. (1999). Constructivismo y aprendizaje significativo. En: F. Díaz y G. Hernández (ed.). Estrategias docentes para un aprendizaje significativo (pp. 13-19). México: McGraw-Hill.
Feixa, C. (2005). La habitación de los adolescentes. Papeles del CEIC, 16, Universidad del País Vasco: Centro de Estudios sobre la Identidad Colectiva. Recuperado de: http:// www.ehu.es/CEIC/papeles/16.pdf

Fonseca, M. (2000). Comunicación oral fundamentos y práctica estratégica. México: Pearson Educación.

González, J.C. (2008). TIC y la transformación de la práctica educativa en el contexto de las sociedades del conocimiento. Revista de Universidad y Sociedad del Conocimiento, $2(5), 1$.

Guitert, M.; Romeu, T. y Pérez, M. (2007). Competencias TIC y trabajo en equipo en entornos virtuales. Revista de Universidad y Sociedad del Conocimiento.

Hannafin, R.D. y Savenye, W.C. (1993). La tecnología en el aula: el nuevo papel del profesor y la resistencia a ella. Tecnología Educativa, 33 (6), 26-31.

Hopenhayn, M. (2002). Educar para la sociedad de la información y de la comunicación. Revista Iberoamericana de Educación, 30.

López, P. (2009). Actitud docente y las TIC. Recuperado de: https://www.scribd.com/doc/ 16302624/Actitud- Docente-y-Las-Tic.

Maldonado, L.F.; Pineda, E.; Quintero, V.J.; Sequeda, J.B.; Lizcano, A.R. (2007). Comunidades de aprendizaje mediadas por redes informáticas. Colombia Educación y Educadores, 11 (1), 199-224.

Marchisio, S.; Plano, M.; Ronco, J. y Von, O. (2005). Combinación de estrategias didácticas e integración de TIC en la enseñanza de fundamentos de física cuántica para ingenieros. V Congreso Internacional Virtual de Educación. Facultad de Ciencias Exactas, Ingeniería y Agrimensura. Argentina: Univ. Nacional de Rosario. 
Martín, D.F.; Lucena, J.H. y Díaz, I.A. (2002). Las actitudes de los docentes hacia la formación en tecnologías de la información y comunicación (TIC) aplicadas a la educación. Contextos educativos, 5, 253-270.

Martínez, P.G. (2013). Estudio de caso sobre la actitud pedagógica del profesor FILEX de la Universidad de Guadalajara hacia las TIC. Ponencia XXI Encuentro de Educación a Distancia, Guadalajara, México. Recuperado de: http://www. udgvirtual.udg.mx/encuentro/anteriores/xxi/ponencias/1 19-174-1-RV.pdf.

Meira, P.A. (2001). La investigación en educación ambiental y las nuevas tecnologías de la información y la comunicación. Teoría de la educación: educación y cultura en la sociedad de la información, 2. [En línea]. gredos.usal.es/ispui/ handle/10366/56437

Meza, A. Pérez, y De la Barreda, B. (2002). Comunidades virtuales de aprendizaje como herramienta didáctica para el apoyo de la labor docente. México. Recuperado de: http://bibliotecadigital.conevyt.org.mx/colecciones/documentos/ somece2002/Grupo3/MezaMezaAdriana 1.pdf.

Ministerio de Educación Nacional (MEN) (2005a). Sistema Educativo Colombiano. Recuperado de: http://www.mineducacion.gov.co/1621/w3-article-233839.html.

Ministerio de Educación Nacional. (2005b). Al tablero 33. Recuperado de: http:// www.mineducacion.gov.co/1621/propertyvalues-31326_tablero_pdf.pdf

Mockus, A. (1984). Movimiento pedagógico y defensa de la calidad de la educación pública. Educación y Cultura, 2, 27-34.

Moreira, M. (2005). Las tecnologías de la información y comunicación en el sistema escolar. Una revisión de las líneas de investigación. Revista Electrónica de Investigación y Evaluación Educativa, 11 (1). Recuperado el 23 de mayo de 2014 de: http://www.uv. es/RELIEVE/v1 1n1/RELIEVEv1 1n1_1.htm

Patiño, M. y Sallé, M. (2013). Las TIC en la enseñanza. Un estudio de casos desde el enfoque biográfico-narrativo. Recuperado de: http://ddd.uab.cat/pub/educar/ educar_a2014m1-6v50n1/educar_a2014m1-6v50n1 p83.pdf.

Sainz Leyva, L. (1998). La comunicación en el proceso pedagógico: algunas reflexiones valorativas. Educación Médica Superior, 12 (1), 26-34.

Salas, R. (2000). La calidad en el desarrollo profesional: avances y desafíos. Educ Med Super. 2(14), 136-147. [En línea]. <http://scielo.sld.cu/scielo.php?script=sci_arttext\&pid=S086421412000000200003\&lng =es\&nrm =iso >

Salinas, J. (2004). Cambios metodológicos con las TIC. Estrategias didácticas y entornos virtuales de enseñanza-aprendizaje. Bordón 56(3-4), 469-481.

Salmerón, H.; Rodríguez, S. y Gutiérrez, C. (2010). Comunicar. Revista Científica Iberoamericana de Comunicación y Educación, 34, 163-17. (Ejemplar dedicado a: Música y pantallas: mediaciones en el nuevo escenario digital). 
Sandholtz, J. D.; Ringstaff, C. y Dwyer, D. (1990). Enseñanza con Tecnología. Ponencia presentada en la Asociación Americana de Investigación Educativa. Boston: Colegio de Profesores de Nueva York.

Tessio, N.; Rumbado, F. y Porro, S. (2006). La formulación de un proyecto de integración de TIC en la formación de formadores. Hacia la hiperlectura en la acción docente. Revista Iberoamericana de Informática Educativa, 4, 21-32.

Tubella, I. (2008). Bajo el asfalto estaba la Red. El País. Recuperado de: http://www.elpais. com/articulo/opinion/asfalto/estaba/Red/ elpepiopi/20080314elpepiopi_13/Tes

Unesco. (2005). Declaración Mundial sobre la Educación Superior. Recuperado de: http:// www.unesco.org/education/educprog/ wche/declaration_spa.htm

Valdés, A.; Arreola, C.; Angulo, J.; Carlos-Martínez, E. y García López, R. (2011). Actitudes de docentes de educación básica hacia las TIC. Magis. Revista Internacional de Investigación en Educación, 3(6), 379-392.

Vence, L. (2011). Uso pedagógico de las TIC para el fortalecimiento de estrategias didácticas del programa Todos a Aprender. Ministerio de Educación Nacional. Recuperado de: http://www.mineducacion.gov.co/ cvn/1665/articles336355_archivo_pdf.pdf.

Verdugo, W. (2004). Actitud docente hacia el uso de la computación. Recuperado de: http://www.comie.org.mx/congreso/ memoriaelectronica/v09/ponencias/at16/ PRE 1178931551 .pdf
Vergara, M. y Castillo, J. E. (2008). Competencias en ciencias: los ambientes digitales SIMAS y Cool Modes. Nómadas, 29, 213-225.

Wiske, M. (1999). La enseñanza para la comprensión. Vinculación entre la investigación y la práctica. Buenos Aires: Paidós.

Zappala, D.; Köppel, A. y Suchodolski, M. (2010). Las TIC en el aula: Estrategias didácticas. Recuperado de: http://escritorioeducacionespecial.educ.ar/datos/recursos/pdf/ propuestas_pedagogicas_capitulo_1.pdf.

Zapata, M. (2006). La actitud de los docentes ante el diseño instruccional tecnológico. Revista de Educación a Distancia, (15). Recuperado de: http://www.redalyc.org/articulo. oa? id $=54701504$. 